Pacific Journal of Mathematics

ANOTHER PROOF OF A THEOREM ON RATIONAL CROSS 


\title{
ANOTHER PROOF OF A THEOREM ON RATIONAL CROSS SECTIONS
}

\author{
MAXWELL ROSENLICHT
}

The extant proofs of the existence of a rational cross section for a transformation space for a connected solvable linear algebraic group either use a certain amount of algebraic curve theory or restrict themselves to the case of a principal space, where the question is one of galois cohomology, the result being equivalent to the statement that $H^{1}(G, k)=0$ for $G$ a $k$-solvable linear algebraic group. The present proof of the general result may be considered more elementary in that it depends only on the standard facts on fields of rationality of algebraic sets.

The result in question says that if $G$ is a k-solvable linear algebraic group and $V$ a transformation space for $G$, all rational over a field $k$, then there exists a $G$-invariant dense $k$-open subset $V^{\prime}$ of $V$ such that $V^{\prime} / G$ exists and is rational over $k$ and a cross section $k$-morphism $V^{\prime} / G \rightarrow V^{\prime}$ exists. This statement appears to be somewhat stronger than the original statement [1, Th. 10], but is exactly equivalent to it (except for the purely technical matter of the possible reducibility of $V$ ) once one accepts the result that any algebraic transformation space admits a quotient space, provided one restricts to a dense open subset [2].

If $V$ happens to be $G$-homogeneous the theorem says nothing more than that $V$ has a point rational over $k$. This special case also implies the general theorem, and that without much labor (in fact the detailed argument in [1] can be much shortened by use of the result quoted at the end of the paragraph above). As for proving the special case just quoted, if one assumes the case $\operatorname{dim} G=1$ there is a straightforward induction argument on $\operatorname{dim} G$ (again refer for details to [1]) so the crux of the proof is that a k-homogeneous space for $G_{a}$ or $G_{m}$ has a point rational over $k$. This note concentrates on the proof of this last statement.

The proof given in [1] that a $k$-homogeneous space for $G_{a}$ or $G_{m}$ has a rational point over $k$ uses some technical information from the theory of algebraic curves, considerably out of the spirit of the present subject. If the homogeneous space happens to be principal homogeneous the question becomes an easy one of galois cohomology [3, pp. 170-171], but some generality has been lost. The present proof is accomplished by starting with a somewhat stronger version of the fact to be proved (Lemma 3 below, which can be taken as the substitute for the Lemma 
to Theorem 10 in [1]), which is easy to prove when $k$ is the universal domain, at the same time retaining some nontrivial content in this extreme case. Lemmas 1 and 2 below are well known, but convenient to have formally stated at this point.

LEMMA 1. The factor group of $G_{a}\left(G_{m}\right)$ by a finite subgroup is isomorphic to $G_{a}\left(G_{m}\right)$.

$G_{a}\left(G_{m}\right)$ is the additive group of the universal domain $\Omega$ (multiplicative group of nonzero elements of $\Omega$ ) with its usual structure as an algebraic set over the prime field. Since $G_{a}$ and $G_{m}$ are commutative we can use induction on the order of the finite subgroup to reduce the proof to the case where this subgroup is cyclic. In the case where the group is $G_{a}$ and $\Omega$ has characteristic $p \neq 0$ the cyclic group generated by a nonzero $a \in \Omega$ is the set of zeros of the polynomial $X^{p}-a^{p-1} X$ and the map sending any $x \in \Omega$ into $x^{p}-a^{p-1} x$ is a separable homomorphism of $G_{a}$ onto itself whose kernel is the cyclic group generated by $a$, proving the lemma in this case. In the remaining cases we do not even need the reduction to the cyclic subgroup: if the group is $G_{a}$ and the field characteristic zero, then $\{0\}$ is the only finite subgroup of $G_{a}$ and there is nothing to prove, while in the case $G_{m}$ a finite subgroup of order $n$ consists of the $n^{\text {th }}$ roots of unity, hence $n$ is prime to the characteristic, and here the map sending each $x \in \Omega$ into $x^{n}$ is a separable homomorphism of $G_{m}$ onto itself with the correct kernel, completing the proof.

LEMMA 2. If $V_{1}, V_{2}$ are homogeneous spaces for the algebraic group $G$ and $\tau: V_{1} \rightarrow V_{2}$ is a rational map such that $\tau\left(g v_{1}\right)=g \tau\left(v_{1}\right)$ (in the sense of rational maps of $G \times V_{1}$ into $V_{2}$ ) then $\tau$ is a morphism.

The proof for this is the usual homogeneity argument: the domain of definition of $\tau$ is a dense open subset of $V_{1}$ that is also $G$-invariant, hence $V_{1}$ itself.

Lemma 3. Let $G$ be the algebraic group $G_{a}$ or $G_{m}$ and let $V$ be a homogeneous space for $G$ of dimension greater than zero, $V$ and the morphism $G \times V \rightarrow V$ biing rational over $k$. Then there exists an algebraic group $G^{\prime}$, rational over $k$ and $k$-isomorphic to $G, a$ surjective k-homomorphism $\varphi: G \rightarrow G^{\prime}$ and a k-isomorphism of algebraic sets $\psi: G^{\prime} \rightarrow V$ such that for any $g \in G$ and $g^{\prime} \in G^{\prime}$ we have $g \psi\left(g^{\prime}\right)=$ $\psi\left(\varphi(g) g^{\prime}\right)$.

We first prove this in the special case where $k$ is the universal domain $\Omega$. The dimension of $V$ is one, and since $G$ is commutative 
each point of $V$ has the same finite isotropy subgroup $H \subset G$. The composition $G \times V \rightarrow V$, which sends $(g, v)$ into $g v$, factors (a priori in a merely set-theoretic sense) into

$$
G \times V \rightarrow(G / H) \times V \rightarrow V
$$

via the natural map $G \rightarrow G / H$. Since the $H$-invariant elements of the function field $\Omega(G \times V)$ can be identified with the elements of $\Omega((G / H) \times V)$, the above map $(G / H) \times V \rightarrow V$ is actually rational. Application of Lemma 2 to the group $G \times G$ operating on $(G / H) \times V$ and $V$ in the appropriate manners shows the map $(G / H) \times V \rightarrow V$ to be a morphism, so that $V$ is homogeneous for $G / H$. By Lemma 1, $G / H$ is isomorphic to $G$, so we are reduced to proving the result when $H=\{e\}$, i.e. all isotropy groups are trivial. Fix a point $v_{0} \in V$. Then the morphism $G \rightarrow V$ which sends any $g \in G$ into $g v_{0}$ is bijective. Identifying the function field $\Omega(V)$ with a subfield of $\Omega(G)$ via this morphism, we see that $\Omega(G)$ is a purely inseparable extension of $Q(V)$. If $x$ is the natural coordinate function on $G$ (so that the map sending any $g \in G$ into $x(g)$ gives the identification of $G$ with $\Omega$ or $\Omega-(0)$ ) then $\Omega(G)=\Omega(x)$ and there exists a positive integer $\nu$ such that $x^{p^{\nu}} \in \Omega(V), p$ being the characteristic of $\Omega$, or 1 if the latter is zero. If $\nu$ is taken minimal then the minimal polynomials of $x$ over $\Omega(V)$ and over $\Omega\left(x^{p^{2}}\right)$ will coincide, giving

$$
[\Omega(G): \Omega(V)]=\left[\Omega(G): \Omega\left(x^{p^{\nu}}\right)\right],
$$

from which it follows that $\Omega(V)=\Omega\left(x^{p^{2}}\right)$. We proceed to prove the lemma in the present case (trivial isotropy groups), taking $G^{\prime}$ to be $G$ and $\varphi$ to be the $p^{\nu}$-th power map. Setting $y=x^{p^{\nu}}$, we have $\Omega(V)=\Omega(y)$ and for $g \in G$ we have $y\left(g v_{0}\right)=(x(g))^{p^{\nu}}$. If we set $v=g^{\prime} v_{0}$, with $g^{\prime} \in G$, and denote by $\circ$ the operation + or $\times$ according as $G$ is $G_{a}$ or $G_{m}$, we have $y(g v)=y\left(g\left(g^{\prime} v_{0}\right)\right)=y\left(\left(g g^{\prime}\right) v_{0}\right)=\left(x\left(g g^{\prime}\right)\right)^{p^{\nu}}=(x(g)) p^{p^{\nu}} \circ\left(x\left(g^{\prime}\right)\right) p^{p^{\nu}}=$ $(x(g))^{p^{\nu}} \circ y(v) . \quad y$ is defined on a dense open subset of $V$, hence, by homogeneity, on all of $V$. If $G=G_{a}$ then $y$ takes on all finite values and if $G=G_{m}$ all finite values except zero, so that $y$ gives an isomorphism between the algebraic set $V$ and $G_{a}$ or $G_{m}$. Everything else we want is trivial to verify, which finishes the case when $k=\Omega$. Now consider the general case. By what we have just proved, there exists a function $y \in \Omega[V]$ inducing an isomorphism (of algebraic sets) between $V$ and $G_{a}$ or $G_{m}$ and a surjective homomorphism $\varphi$ from $G$ into $G_{a}$ or $G_{m}$ (identified with $\Omega$ or $\Omega-(0)$, so that $\varphi$ can be regarded as a numerical function) such that $y(g v)=\varphi(g) \circ y(v)$ for all $g \in G, v \in V$, and we need only show that $y$ and $\varphi$ may be taken to be rational over $k$. So express $y$ and $\varphi$ as linear combinations with coefficients in $\Omega$ of elements of $k[V]$ and $k[G]$ respectively. By linear algebra we may write 


$$
y=\sum_{i=1}^{n} c_{i} y_{i}, \quad \varphi=\sum_{i=1}^{n} c_{i} \varphi_{i},
$$

where $\left\{c_{i}\right\} \subset \Omega$ are linearly independent over $k$ and each $y_{i} \in k[V]$, each $\varphi_{i} \in k[G]$. If $G=G_{a}$, then $y(g v)=\varphi(g)+y(v)$, so

$$
\sum_{i=1}^{n} c_{i} y_{i}(g v)=\sum_{i=1}^{n} c_{i} \varphi_{i}(g)+\sum_{i=1}^{n} c_{i} y_{i}(v),
$$

or

$$
\sum_{i=1}^{n} c_{i}\left(y_{i}(g v)-\varphi_{i}(g)-y_{i}(v)\right)=0 .
$$

Since $G \times V$ is rational over $k, k(G \times V)$ and $\Omega$ are linearly disjoint over $k$, so $y_{i}(g v)=\varphi_{i}(g)+y_{i}(v)$ for all $i$. Each $y_{i} \in \Omega[y]$ so there exists $f_{i}(Y) \in \Omega[Y]$ ( $Y$ an indeterminate over $\Omega$ ) such that $y_{i}=f_{i}(y)$, giving

$$
f_{i}(\varphi(g)+y(v))=\varphi_{i}(g)+f_{i}(y(v)) .
$$

Now if $x \in k[G]$ is the additive coordinate function on $G_{a}$ then $\varphi_{i} \in k[G]=$ $k[x]$ has a certain degree $\nu_{i}$ in $x$ and $\varphi=\sum_{i} c_{i} \varphi_{i}$ has degree equal to $\max _{i} \nu_{i}>0$, so the last equation implies that the degree of each $f_{i}$ is at most one. Not all $f_{i}$ 's are constant, so say $f_{1}$ is nonconstant, whence $f_{1}(Y)=a Y+b$, with $a, b \in \Omega, a \neq 0$. Then $y_{1}=a y+b$. We can now replace $y$ by $y_{1}$ as a coordinate function on $V$ (at the same time changing $\varphi$ appropriately), i.e. we can assume that $y \in k[V]$. Then the equation $\varphi(g)=y(g v)-y(v)$ gives $\varphi(g)=y(g \circ 0)-y(0)$, so $\varphi \in k[G]$, completing the proof for the $G_{a}$ case. Finally let $G=G_{m}$. Then $y(g v)=\varphi(g) y(v)$, so

$$
\sum_{i=1}^{n} c_{i} y_{i}(g v)=\sum_{i=1}^{n} c_{i} \varphi(g) y_{i}(v) .
$$

But $\varphi$ is a surjective homomorphism from $G_{m}$ into itself, therefore given by sending any $x$ into $x^{\nu}$ for some nonzero integer $\nu$, in particular $\varphi \in k[G]$. Thus linear disjointness implies that $y_{i}(g v)=\varphi(g) y_{i}(v)$ for all $i$. In the present case $\Omega[V]=\Omega[y, 1 / y]$ so we can write $y_{i}=$ $\sum_{j} a_{i j} y^{j}$, where each $a_{i j} \in \Omega$ and the index $j$ ranges over a finite set of integers, some possibly negative. We therefore have

$$
\sum_{j} a_{i j}(\varphi(g) y(v))^{j}=\varphi(g) \sum_{j} a_{i j}(y(v))^{j}
$$

for all $g \in G, v \in V$, which implies that $a_{i j}=0$ if $j \neq 1$. Thus $y_{i}=a_{i} y$, with $a_{i} \in \Omega$. If $a_{1} \neq 0$ we can replace $y$ by $y_{1}$ as a coordinate function on $V$ so as to get, once more, $y \in k[V]$, and we are done. 


\section{REFERENCES}

1. M. Rosenlicht, Some basic theorems on algebraic groups, Amer. J. Math. 78 (1956), 401-443.

2. - A remark on quotient spaces, Anais da Academia Brasileira de Ciencias 35 (1963), 487-489.

3. J-P. Serre, Groupes algébriques et corps de classes, Hermann, Paris, 1959.

Received February 6, 1966. Research supported by National Science Foundation grant number GT-3990.

University of California, Berkeley 



\section{PACIFIC JOURNAL OF MATHEMATICS}

H. SAMELSON

Stanford University

Stanford, California

J. P. JaNs

University of Washington

Seattle, Washington 98105

\section{EDITORS}

J. DugundJI

University of Southern California Los Angeles, California 90007

RICHARD ARENS

University of California

Los Angeles, California 90024

\section{ASSOCIATE EDITORS}

E. F. BECKENBACH

B. H. NEUMANN

F. WOLF

K. YosidA

\section{SUPPORTING INSTITUTIONS}

UNIVERSITY OF BRITISH COLUMBIA

CALIFORNIA INSTITUTE OF TECHNOLOGY

UNIVERSITY OF CALIFORNIA

MONTANA STATE UNIVERSITY

UNIVERSITY OF NEVADA

NEW MEXICO STATE UNIVERSITY

OREGON STATE UNIVERSITY

UNIVERSITY OF OREGON

OSAKA UNIVERSITY

UNIVERSITY OF SOUTHERN CALIFORNIA
STANFORD UNIVERSITY

UNIVERSITY OF TOKYO

UNIVERSITY OF UTAH

WASHINGTON STATE UNIVERSITY

UNIVERSITY OF WASHINGTON

AMERICAN MATHEMATICAL SOCIETY CHEVRON RESEARCH CORPORATION TRW SYSTEMS

NAVAL ORDNANCE TEST STATION 


\section{Pacific Journal of Mathematics}

\section{Vol. 20, No. $1 \quad$ September, 1967}

Leonard Daniel Baumert, Extreme copositive quadratic forms. II ........ 1

Edward Lee Bethel, A note on continuous collections of disjoint

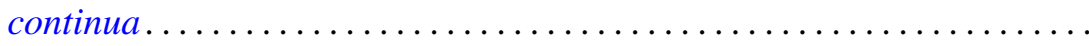

Delmar L. Boyer and Adolf G. Mader, A representation theorem for abelian groups with no elements of infinite p-height ...................

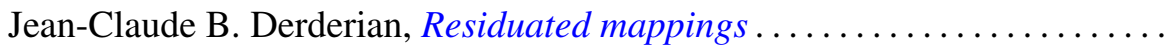

Burton I. Fein, Representations of direct products of finite groups ......... 45

John Brady Garnett, A topological characterization of Gleason parts.......

Herbert Meyer Kamowitz, On operators whose spectrum lies on a circle or

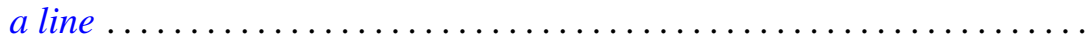

Ignacy I. Kotlarski, On characterizing the gamma and the normal distribution ........................................

Yu-Lee Lee, Topologies with the same class of homeomorphisms ..........

Moshe Mangad, Asymptotic expansions of Fourier transforms and discrete polyharmonic Green's functions ...........................

Jürg Thomas Marti, On integro-differential equations in Banach spaces ....

Walter Philipp, Some metrical theorems in number theory.............. 109

Maxwell Alexander Rosenlicht, Another proof of a theorem on rational

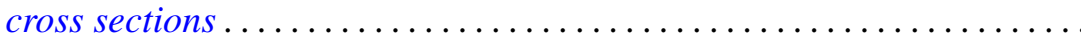

Kenneth Allen Ross and Karl Robert Stromberg, Jessen's theorem on Riemann sums for locally compact groups

Stephen Simons, A theorem on lattice ordered groups, results of Ptak, Namioka and Banach, and a front-ended proof of Lebesgue's theorem...

Morton Lincoln Slater, On the equation $\varphi(x)=\int_{x} x+1 K(\xi) f[\varphi(\xi)] d \xi \ldots$ 155 Arthur William John Stoddart, Existence of optimal controls .. 167 Burnett Roland Toskey, A system of canonical forms for rings on a direct sum of two infinite cyclic groups ....................

Jerry Eugene Vaughan, A modification of Morita's characterization of dimension 